\title{
Reliability Impacts in Life Support Architecture and Technology Selection
}

\begin{abstract}
Equivalent System Mass (ESM) and reliability estimates were performed for different life support architectures based primarily on International Space Station (ISS) technologies. The analysis was applied to a hypothetical 1-year deep-space mission. High-level fault trees were initially developed relating loss of life support functionality to the Loss of Crew (LOC) top event. System reliability was then expressed as the complement (nonoccurrence) this event and was increased through the addition of redundancy and spares, which added to the ESM. The reliability analysis assumed constant failure rates and used current projected values of the Mean Time Between Failures (MTBF) from an ISS database where available. Results were obtained showing the dependence of ESM on system reliability for each architecture.
\end{abstract}

Although the analysis employed numerous simplifications and many of the input parameters are considered to have high uncertainty, the results strongly suggest that achieving necessary reliabilities for deep-space missions will add substantially to the life support system mass. As a point of reference, the reliability for a single-string architecture using the most regenerative combination of ISS technologies without unscheduled replacement spares was estimated to be less than $1 \%$. The results also demonstrate how adding technologies in a serial manner to increase system closure forces the reliability of other life support technologies to increase in order to meet the system reliability requirement. This increase in reliability results in increased mass for multiple technologies through the need for additional spares. Alternative parallel architecture approaches and approaches with the potential to "do more with less" are discussed. The "tall poles" in life support ESM are also reexamined in light of estimated reliability impacts.

\section{Authors:}

Kevin Lange

Jacobs Technology

(281) $461-5388$

Molly Anderson

NASA/JSC/EC2

(281) 483-9149 